PROCEEDINGS OF THE

AMERICAN MATHEMATICAL SOCIETY

Volume 132, Number 7, Pages 2127-2132

S 0002-9939(04)07310-1

Article electronically published on January 23, 2004

\title{
TOTAL CURVATURES OF A CLOSED CURVE IN EUCLIDEAN $n$-SPACE
}

\author{
L. HERNÁNDEZ ENCINAS AND J. MUÑOZ MASQUÉ
}

(Communicated by Jon G. Wolfson)

\begin{abstract}
A classical result by J. W. Milnor states that the total curvature of a closed curve $C$ in the Euclidean $n$-space is the limit of the total curvatures of polygons inscribed in $C$. In the present paper a similar geometric interpretation is given for all total curvatures $\int_{C}\left|\kappa_{r}\right| \mathrm{d} s, r=1, \ldots, n-1$.
\end{abstract}

\section{Preliminaries and Statement of the main Result}

A curve $\mathbf{x}(t)$ in $\mathbb{R}^{n}$ defined on an interval $t \in I$ and of class $C^{k}$ is said to be in general position up to order $r, 1 \leq r \leq n$, at $t_{0}$ if $k \geq r$ and the derivatives $\mathbf{x}^{\prime}\left(t_{0}\right), \mathbf{x}^{\prime \prime}\left(t_{0}\right), \ldots, \mathbf{x}^{(r)}\left(t_{0}\right)$ are linearly independent. If $k>r \geq 1$, then the curves in general position up to order $r$ at every $t \in I$ are a dense open subset in $C^{k}\left(I, \mathbb{R}^{n}\right)$ with respect to the strong topology for $1 \leq r \leq n-1$, but curves in general position up to order $n$ are not dense since inflection points cannot be eliminated by small perturbations. Because of this we say that a curve in $\mathbb{R}^{n}$ is in general position if it is so up to order $n-1$ and its class is $\geq n$. If $\mathbf{x}(t)$ is a closed curve in general position up to first order (i.e., $\mathbf{x}: \mathbb{R} \rightarrow \mathbb{R}^{n}$ is a periodic immersion), then the arc length $s(t)=\int_{0}^{t}\left|\mathbf{x}^{\prime}(t)\right| \mathrm{d} t$ satisfies $s(t+\omega)=s(t)+l, \forall t$, where $\omega$ is a period (i.e., $\omega>0, \mathbf{x}(t+\omega)=\mathbf{x}(t), \forall t)$ and $l=\int_{0}^{\omega}\left|\mathbf{x}^{\prime}(t)\right| \mathrm{d} t$ is the length of the curve.

Curves in general position admit a Frénet frame; that is, if $\mathbf{x}$ is parametrized by its arc length $s$, there exist vectors $\mathbf{f}_{1}(s), \ldots, \mathbf{f}_{n}(s)$ and functions $\kappa_{1}(s), \ldots, \kappa_{n-1}(s)$ such that:

(a) $\mathbf{f}_{i}\left(\right.$ resp. $\left.\kappa_{i}\right)$ is of class $\geq n-i$ (resp. $\geq n-1-i$ ) for $1 \leq i \leq n-1$, $\mathbf{f}_{n}$ is of class $\geq 1$ and $\kappa_{i}>0$ for $1 \leq i \leq n-2$.

(b) $\left(\mathbf{f}_{1}(s), \ldots, \mathbf{f}_{n}(s)\right)$ is a positively oriented orthonormal basis for every $s \in$ $[0, l]$.

(c) The following formulas hold true:

(c.1) $\mathrm{d} \mathbf{x} / \mathrm{d} s=\mathbf{f}_{1}$,

(c.2) $\mathrm{d} \mathbf{f}_{1} / \mathrm{d} s=\kappa_{1} \mathbf{f}_{2}$,

(c.3) $\mathrm{d} \mathbf{f}_{i} / \mathrm{d} s=-\kappa_{i-1} \mathbf{f}_{i-1}+\kappa_{i} \mathbf{f}_{i+1}$ for $2 \leq i \leq n-1$,

(c.4) $\mathrm{d} \mathbf{f}_{n} / \mathrm{d} s-\kappa_{n-1} \mathbf{f}_{n-1}$.

Received by the editors February 26, 2003 and, in revised form, March 25, 2003.

2000 Mathematics Subject Classification. Primary 53A04; Secondary 28A75, 51M20.

Key words and phrases. Curvatures of a curve, Frénet frame, polygon, total curvature.

This work was supported by Ministerio de Ciencia y Tecnología (Spain) under grants TIC20010586 and BFM2002-00141. 
Given a finite number of vectors $\mathbf{v}_{1}, \ldots, \mathbf{v}_{k}$ in $\mathbb{R}^{n}$, we denote by $\left\langle\mathbf{v}_{1}, \ldots, \mathbf{v}_{k}\right\rangle$ (resp. $\left[\mathbf{v}_{1}, \ldots, \mathbf{v}_{k}\right]$ ) the vector (resp. affine) subspace that they span. We have $\operatorname{dim}\left[\mathbf{v}_{1}, \ldots, \mathbf{v}_{k}\right]=\operatorname{dim}\left\langle\mathbf{v}_{1}-\mathbf{v}_{i}, \ldots, \hat{\mathbf{v}}_{i}, \ldots, \mathbf{v}_{k}-\mathbf{v}_{i}\right\rangle$ for every $1 \leq i \leq k$.

We extend the scalar product in $\mathbb{R}^{n}$ to $\wedge^{r} \mathbb{R}^{n}$ by imposing

$$
\left\langle\mathbf{v}_{1} \wedge \ldots \wedge \mathbf{v}_{r}, \mathbf{w}_{1} \wedge \ldots \wedge \mathbf{w}_{r}\right\rangle=\operatorname{det}\left(\left\langle\mathbf{v}_{i}, \mathbf{w}_{j}\right\rangle\right)_{i, j=1, \ldots, r}
$$

We have

i) $\left\|\mathbf{v}_{1} \wedge \ldots \wedge \mathbf{v}_{r}\right\| \leq\left\|\mathbf{v}_{1}\right\| \cdots\left\|\mathbf{v}_{r}\right\|$, and

ii) if $\left(\mathbf{v}_{1}, \ldots, \mathbf{v}_{r}\right)$ is an orthonormal system, then $\mathbf{v}_{1} \wedge \ldots \wedge \mathbf{v}_{r}$ is a unitary vector.

We recall that the first $n-1$ vectors $\mathbf{f}_{1}(s), \ldots, \mathbf{f}_{n-1}(s)$ in the Frénet frame of a curve $\mathbf{x}(s)$ are obtained by applying the Gram-Schmidt process ([4, 2.1.9]) to the system $\mathbf{x}^{\prime}(s), \mathbf{x}^{\prime \prime}(s), \ldots, \mathbf{x}^{(n-1)}(s)$; hence we have

1) $\left\langle\mathbf{f}_{1}(s), \ldots, \mathbf{f}_{r}(s)\right\rangle=\left\langle\mathbf{x}^{\prime}(s), \ldots, \mathbf{x}^{(r)}(s)\right\rangle$,

2) $\left(\mathbf{f}_{1}(s), \ldots, \mathbf{f}_{r}(s)\right)$ and $\left(\mathbf{x}^{\prime}(s), \ldots, \mathbf{x}^{(r)}(s)\right)$ define the same orientation, for all $0 \leq s \leq l, 1 \leq r \leq n-1$.

A closed polygon in $\mathbb{R}^{n}$ is a finite sequence of points $\Pi=\left(\mathbf{p}_{0}, \mathbf{p}_{1}, \ldots, \mathbf{p}_{m-1}, \mathbf{p}_{m}\right)$ such that $\mathbf{p}_{m}=\mathbf{p}_{0}$. We take the subindices of the points in $\Pi$ modulo $m$ (e.g. $\mathbf{p}_{m+3}=\mathbf{p}_{3}$, etc.), so that cyclic permutations are allowed in writing the vertices of П. A polygon is said to be in general position up to order $r, 1 \leq r \leq n$, if any system of $r+1$ consecutive vertices of the polygon spans an affine subspace of dimension $r$; that is, $\operatorname{dim}\left[\mathbf{p}_{i}, \mathbf{p}_{i+1}, \ldots, \mathbf{p}_{i+r}\right]=r$ for every $i$. As in the case of curves, a closed polygon in $\mathbb{R}^{n}$ is in general position if it is so up to order $n-1$.

If $\Pi=\left(\mathbf{x}_{0}, \ldots, \mathbf{x}_{m}\right)$ is a closed polygon inscribed in a closed curve $C: \mathbf{x}(t)$, i.e., $\mathbf{x}_{i} \mathbf{x}\left(t_{i}\right), 0 \leq i \leq m, t_{0}<\ldots<t_{m}$, we set $|\Pi|=\max _{i}\left|t_{i+1}-t_{i}\right|$. If $C$ is in general position up to order $r$, then there exists $\varepsilon>0$ such that every closed polygon $\Pi$ inscribed in $C$ with $|\Pi|<\varepsilon$ is also in general position up to order $r$. Assume $m \geq r+2$. Let us denote by $0 \leq \alpha_{i} \leq \pi$ the angle determined by the affine subspaces $\left[\mathbf{x}_{i}, \mathbf{x}_{i+1}, \ldots, \mathbf{x}_{i+r}\right]$ and $\left[\mathbf{x}_{i+1}, \mathbf{x}_{i+2}, \ldots, \mathbf{x}_{i+r}, \mathbf{x}_{i+r+1}\right]$; i.e., $\alpha_{i}=\measuredangle\left(\mathbf{u}_{i}, \mathbf{u}_{i+1}\right)$, where $\mathbf{u}_{i}=\left(\mathbf{x}_{i+1}-\mathbf{x}_{i}\right) \wedge \ldots \wedge\left(\mathbf{x}_{i+r}-\mathbf{x}_{i+r-1}\right)$. The $r$-th total curvature of $\Pi$ is defined by

$$
T^{r}(\Pi)=\sum_{i=0}^{m-1} \alpha_{i}
$$

We have

Theorem 1.1. If $C: \mathbf{x}(s)$ is a closed curve in general position in $\mathbb{R}^{n}$ parametrized by its arc length and $\Pi$ is a closed polygon inscribed in $C$, then we have

$$
\lim _{|\Pi| \rightarrow 0} T^{r}(\Pi)=\int_{C}\left|\kappa_{r}(s)\right| \mathrm{d} s, \quad 1 \leq r \leq n-1 .
$$

This result provides a geometric interpretation of curvatures of a curve, especially interesting for higher orders. We also remark that, unlike the case $r=1$, for $2 \leq r \leq n-1$, Lemma 1.1 in [3] does not hold as numerical experiments show. However, $\int_{C}\left|\kappa_{r}(s)\right| \mathrm{d} s$ is the least upper bound of the values $T^{r}(\Pi)$ for all closed polygons $\Pi$ inscribed in $C$. In fact, given such a polygon $\Pi$, it is not difficult to construct a sequence of inscribed closed polygons $\Pi_{h}$ such that $\Pi_{1}=\Pi$ and $T^{r}\left(\Pi_{1}\right) \leq T^{r}\left(\Pi_{2}\right) \leq \ldots \leq T^{r}\left(\Pi_{h}\right) \leq \ldots$, and we apply the result above.

In the particular case of the torsion of a curve in a three-dimensional Euclidean space, the result above is also given in [1, Theorem 8.2.3] by using a completely 
different technique. We thank the referee of the present paper who mentioned that reference to us.

\section{TWO BASIC LEMMAS}

Lemma 2.1. Set $a_{h k}=\frac{1}{h !}\left(\Delta_{k} s_{i}\right)^{h} ; \lambda_{i}=\operatorname{det}\left(a_{h k}\right), h, k=1, \ldots, r ; \mu_{i}=\operatorname{det}\left(a_{h k}\right)$, $1 \leq h \leq r+1, h \neq r, 1 \leq k \leq r ; \mathbf{x}_{i}^{(j)}=\mathrm{d}^{j} \mathbf{x} / \mathrm{d} s^{j}\left(s_{i}\right)$, and

$$
\mathbf{v}_{i}=\mathbf{u}_{i}-\lambda_{i} \mathbf{x}_{i}^{(1)} \wedge \ldots \wedge \mathbf{x}_{i}^{(r)}-\mu_{i} \mathbf{x}_{i}^{(1)} \wedge \ldots \wedge \mathbf{x}_{i}^{(r-1)} \wedge \mathbf{x}_{i}^{(r+1)} .
$$

For every $\varepsilon>0$, there exists $\delta>0$ such that $|\Pi|<\delta$ implies $\left\|\mathbf{v}_{i}\right\|<\varepsilon\left(\Delta_{r} s_{i}\right)^{1+\left(\begin{array}{c}r+1 \\ 2\end{array}\right)}$.

Proof. By using Taylor's formula with integral remainder (e.g., see [2, X, §8]) and expanding $\mathbf{x}(s)$ at $s=s_{i}$ up to order $r$ we have

$$
\mathbf{x}(s)-\mathbf{x}_{i}=\sum_{j=1}^{r} \frac{\left(s-s_{i}\right)^{j}}{j !} \mathbf{x}_{i}^{(j)}+\left(s-s_{i}\right)^{r+1} \mathbf{r}\left(s_{i}, s\right),
$$

where $(u, v) \mapsto \mathbf{r}(u, v)$ is a continuous map such that $\mathbf{r}(s, s)=\frac{1}{(r+1) !} \mathbf{x}^{(r+1)}(s)$. Evaluating $\mathbf{x}(s)-\mathbf{x}_{i}$ at $s_{i+1}, \ldots, s_{i+r}$ and taking into account that $\mathbf{u}_{i}$ can be written as $\left(\mathbf{x}_{i+1}-\mathbf{x}_{i}\right) \wedge\left(\mathbf{x}_{i+2}-\mathbf{x}_{i}\right) \wedge \ldots \wedge\left(\mathbf{x}_{i+r}-\mathbf{x}_{i}\right)$, we obtain

$$
\begin{aligned}
\mathbf{u}_{i}=\left(\sum_{j_{1}=1}^{r} \frac{\left(\Delta_{1} s_{i}\right)^{j_{1}}}{j_{1} !} \mathbf{x}_{i}^{\left(j_{1}\right)}+\left(\Delta_{1} s_{i}\right)^{r+1} \mathbf{r}_{i, i+1}\right) & \wedge \ldots \\
& \wedge\left(\sum_{j_{r}=1}^{r} \frac{\left(\Delta_{r} s_{i}\right)^{j_{r}}}{j_{r} !} \mathbf{x}_{i}^{\left(j_{r}\right)}+\left(\Delta_{r} s_{i}\right)^{r+1} \mathbf{r}_{i, i+r}\right),
\end{aligned}
$$

where we have set $\mathbf{r}_{i j}=\mathbf{r}\left(s_{i}, s_{j}\right)$. Expanding the $r$ exterior products in the formula above, we can sort the terms thus obtained according to the number of remainder factors that they contain. Those containing no remainder factor are of the form

$$
\frac{\left(\Delta_{1} s_{i}\right)^{j_{1}}}{j_{1} !} \cdots \frac{\left(\Delta_{r} s_{i}\right)^{j_{r}}}{j_{r} !} \mathbf{x}_{i}^{\left(j_{1}\right)} \wedge \ldots \wedge \mathbf{x}_{i}^{\left(j_{r}\right)}, \quad\left\{j_{1}, \ldots, j_{r}\right\}=\{1, \ldots, r\} .
$$

Hence the contribution of these terms is $\lambda_{i} \mathbf{x}_{i}^{(1)} \wedge \ldots \wedge \mathbf{x}_{i}^{(r)}$. The terms containing one factor of the remainder exactly are as follows:

$$
\begin{aligned}
& \frac{\left(\Delta_{1} s_{i}\right)^{j_{1}}}{j_{1} !} \cdots \underbrace{\left(\Delta_{p} s_{i}\right)^{r+1}}_{p} \cdots \frac{\left(\Delta_{r} s_{i}\right)^{j_{r}}}{j_{r} !} \mathbf{x}_{i}^{\left(j_{1}\right)} \wedge \ldots \wedge \underbrace{\mathbf{r}_{i, i+p}}_{p} \wedge \ldots \wedge \mathbf{x}_{i}^{\left(j_{r}\right)} \\
& =\frac{\left(\Delta_{1} s_{i}\right)^{j_{1}}}{j_{1} !} \cdots \underbrace{\left(\Delta_{p} s_{i}\right)^{r+1}}_{p} \cdots \frac{\left(\Delta_{r} s_{i}\right)^{j_{r}}}{j_{r} !} \frac{1}{(r+1) !} \mathbf{x}_{i}^{\left(j_{1}\right)} \wedge \ldots \wedge \underbrace{\mathbf{x}_{i}^{(r+1)}}_{p} \wedge \ldots \wedge \mathbf{x}_{i}^{\left(j_{r}\right)} \\
& +\frac{\left(\Delta_{1} s_{i}\right)^{j_{1}}}{j_{1} !} \cdots \underbrace{\left(\Delta_{p} s_{i}\right)^{r+1}}_{p} \cdots \frac{\left(\Delta_{r} s_{i}\right)^{j_{r}}}{j_{r} !} \mathbf{x}_{i}^{\left(j_{1}\right)} \wedge \ldots \wedge \underbrace{\left(\mathbf{r}_{i, i+p}-\mathbf{r}_{i i}\right)}_{p} \wedge \ldots \wedge \mathbf{x}_{i}^{\left(j_{r}\right)},
\end{aligned}
$$

where $\left\{j_{1}, \ldots, \hat{j_{p}}, \ldots, j_{r}\right\}$ is equal to one of the following $r$ sets: $\{1,2, \ldots, r-1\}$, $\{1,2, \ldots, r-2, r\},\{1,2, \ldots, r-3, r-1, r\}, \ldots,\{2,3, \ldots, r\}$. We have

$$
\begin{aligned}
j_{1}+\ldots+\hat{j_{p}}+\ldots+j_{r}+(r+1)=1 & +\left(\begin{array}{c}
r+1 \\
2
\end{array}\right), \\
& \text { if }\left\{j_{1}, \ldots, \widehat{j_{p}}, \ldots, j_{r}\right\}=\{1,2, \ldots, r-1\},
\end{aligned}
$$




$$
\begin{aligned}
& j_{1}+\ldots+\widehat{j_{p}}+\ldots+j_{r}+(r+1) \geq 2+\left(\begin{array}{c}
r+1 \\
2
\end{array}\right), \\
& \text { if }\left\{j_{1}, \ldots, \widehat{j_{p}}, \ldots, j_{r}\right\} \neq\{1,2, \ldots, r-1\} .
\end{aligned}
$$

Collecting the first summands of the right-hand side in the previous equation for $\left\{j_{1}, \ldots, \widehat{j_{p}}, \ldots, j_{r}\right\}=\{1,2, \ldots, r-1\}$ we obtain $\mu_{i} \mathbf{x}_{i}^{(1)} \wedge \ldots \wedge \mathbf{x}_{i}^{(r-1)} \wedge \mathbf{x}_{i}^{(r+1)}$. Since $\Delta_{j} s_{i} \leq j|\Pi|$, and $|\Pi|<\delta$ implies $\left\|\mathbf{r}_{i, i+p}-\mathbf{r}_{i i}\right\|<\varepsilon$ for small enough $\delta$, the lemma is proven.

Lemma 2.2. With the same notation as above, set

$$
\begin{aligned}
& s_{i}^{\prime}=\frac{s_{i}+s_{i+1}+\ldots+s_{i+r}}{r+1}, \quad 0 \leq i \leq m-1, \\
& \theta_{i}=\measuredangle\left(\mathbf{u}_{i},\left(\mathbf{f}_{1} \wedge \ldots \wedge \mathbf{f}_{r}\right)\left(s_{i}^{\prime}\right)\right) .
\end{aligned}
$$

Then, for every $\varepsilon>0$, there exists $\delta>0$ such that $|\Pi|<\delta$ implies $\theta_{i}<\left(\Delta_{r} s_{i}\right) \varepsilon$, where $\Delta_{j} s_{i}=s_{i+j}-s_{i}$.

Proof. First of all, as a computation shows, we have

$$
\begin{gathered}
\lambda_{i}=\frac{\Delta_{1} s_{i} \cdots \Delta_{r} s_{i}}{1 ! 2 ! \cdots r !} \cdot \text { Vandermonde }\left(\Delta_{1} s_{i}, \ldots, \Delta_{r} s_{i}\right) \\
=\frac{\Delta_{1} s_{i} \cdots \Delta_{r} s_{i}}{1 ! 2 ! \cdots r !} \prod_{1 \leq h<k \leq r}\left(s_{i+k}-s_{i+h}\right) \\
\mu_{i}=\frac{\Delta_{1} s_{i} \cdots \Delta_{r} s_{i}}{1 ! 2 ! \cdots(r-1) !(r+1) !}\left|\begin{array}{cccc}
1 & 1 & \ldots & 1 \\
\Delta_{1} s_{i} & \Delta_{2} s_{i} & \ldots & \Delta_{r} s_{i} \\
\vdots & \vdots & \ddots & \vdots \\
\left(\Delta_{1} s_{i}\right)^{r-2} & \left(\Delta_{2} s_{i}\right)^{r-2} & \ldots & \left(\Delta_{r} s_{i}\right)^{r-2} \\
\left(\Delta_{1} s_{i}\right)^{r} & \left(\Delta_{2} s_{i}\right)^{r} & \ldots & \left(\Delta_{r} s_{i}\right)^{r}
\end{array}\right| \\
=\lambda_{i} \frac{\Delta_{1} s_{i}+\ldots+\Delta_{r} s_{i}}{r+1} \lambda_{i} \frac{s_{i+1}+\ldots+s_{i+r}-r s_{i}}{r+1}=\lambda_{i}\left(s_{i}^{\prime}-s_{i}\right) .
\end{gathered}
$$

Moreover, from the formulas (c.1)-(c.4) we obtain $\mathbf{x}^{(j)} \sum_{i=1}^{j} f_{i j} \mathbf{f}_{i}, 1 \leq j \leq n$, where the functions $f_{i j}$ satisfy the following recurrence relations: $f_{11}=1, f_{12}=0$, $f_{22}=\kappa_{1}$, and for $3 \leq j \leq n$,

$$
\begin{aligned}
f_{1 j} & =f_{1, j-1}^{\prime}-\kappa_{1} f_{2, j-1}, & & i=1, \\
f_{i j} & =f_{i, j-1}^{\prime}+\kappa_{i-1} f_{i-1, j-1}-\kappa_{i} f_{i+1, j-1}, & & 2 \leq i \leq j-2, \\
f_{j-1, j} & =f_{j-1, j-1}^{\prime}+\kappa_{j-2} f_{j-2, j-1}, & & i=j-1, \\
f_{j j} & =\kappa_{j-1} f_{j-1, j-1}, & & i=j .
\end{aligned}
$$

Hence, letting $p_{j}=f_{11} \cdots f_{j j}$, from the formulas above we obtain

$$
\begin{aligned}
\mathbf{x}^{(1)} \wedge \ldots \wedge \mathbf{x}^{(r)} & =p_{r} \mathbf{f}_{1} \wedge \ldots \wedge \mathbf{f}_{r} \\
\mathbf{x}^{(1)} \wedge \ldots \wedge \mathbf{x}^{(r-1)} \wedge \mathbf{x}^{(r+1)} & =p_{r-1}\left(f_{r r}^{\prime}+\kappa_{r-1} f_{r-1, r}\right) \mathbf{f}_{1} \wedge \ldots \wedge \mathbf{f}_{r} \\
& +\kappa_{r} p_{r} \mathbf{f}_{1} \wedge \ldots \wedge \mathbf{f}_{r-1} \wedge \mathbf{f}_{r+1} \\
& =\left(p_{r} \mathbf{f}_{1} \wedge \ldots \wedge \mathbf{f}_{r}\right)^{\prime}
\end{aligned}
$$


as follows taking into account that $p_{j}^{\prime}=p_{j-1}\left(f_{j j}^{\prime}+\kappa_{j-1} f_{j-1, j}\right), 2 \leq j \leq n$. Expanding the function $p_{r} \mathbf{f}_{1} \wedge \ldots \wedge \mathbf{f}_{r}$ on the interval $\left[s_{i}, s_{i}^{\prime}\right]$ up to first order with remainder $R$, from Lemma 2.1 and the value for $\mu_{i}$ above, we have

$\mathbf{u}_{i}=\lambda_{i}\left[\left(p_{r} \mathbf{f}_{1} \wedge \ldots \wedge \mathbf{f}_{r}\right)\left(s_{i}^{\prime}\right)-\left(s_{i}^{\prime}-s_{i}\right)^{2} R\left(s_{i}, s_{i}^{\prime}\right)\right]+\mathbf{v}_{i}=\lambda_{i}\left(p_{r} \mathbf{f}_{1} \wedge \ldots \wedge \mathbf{f}_{r}\right)\left(s_{i}^{\prime}\right)+\mathbf{v}_{i}^{\prime}$,

where $\left\|\mathbf{v}_{i}^{\prime}\right\|<\varepsilon\left(\Delta_{r} s_{i}\right)^{1+\left(\begin{array}{c}r+1 \\ 2\end{array}\right)}$ for small enough $|\Pi|$. Letting $\overline{\mathbf{u}}_{i}=\mathbf{u}_{i} /\left(\lambda_{i} p_{r}\left(s_{i}^{\prime}\right)\right)$, we have $\theta_{i} \measuredangle\left(\overline{\mathbf{u}}_{i},\left(\mathbf{f}_{1} \wedge \ldots \wedge \mathbf{f}_{r}\right)\left(s_{i}^{\prime}\right)\right)$.

Taking into account that $\lambda_{i} \leq \frac{1}{1 ! 2 ! \cdots r !}\left(\Delta_{r} s_{i}\right)^{\left(\begin{array}{c}r+1 \\ 2\end{array}\right)}$, and $\min p_{r}(s)>0$ since $C$ is in general position, we obtain $\left\|\overline{\mathbf{u}}_{i}-\left(\mathbf{f}_{1} \wedge \ldots \wedge \mathbf{f}_{r}\right)\left(s_{i}^{\prime}\right)\right\|<c \varepsilon \Delta_{r} s_{i}$, where $c>0$ is a constant. Hence

$$
\cos \theta_{i}>\frac{\left\|\overline{\mathbf{u}}_{i}\right\|^{2}+1-c^{2} \varepsilon^{2}\left(\Delta_{r} s_{i}\right)^{2}}{2\left\|\overline{\mathbf{u}}_{i}\right\|} \geq \sqrt{1-c^{2} \varepsilon^{2}\left(\Delta_{r} s_{i}\right)^{2}}
$$

and accordingly $\left|\sin \theta_{i}\right|<c \varepsilon \Delta_{r} s_{i}$, thus proving Lemma 2.2 .

\section{Proof of Theorem 1.1}

Let $S\left(\bigwedge^{r} \mathbb{R}^{n}\right)$ be the unit sphere in $\bigwedge^{r} \mathbb{R}^{n}$. We remark that the spherical distance between two points $\mathbf{x}, \mathbf{y}$ is equal to $\measuredangle(\mathbf{x}, \mathbf{y})$, and the length of a spherical curve is the limit of the lengths of the geodesic polygons inscribed on it. Let us apply these properties to the spherical curve $s \mapsto\left(\mathbf{x}_{1} \wedge \ldots \wedge \mathbf{x}_{r}\right)(s)$ and to the geodesic polygon $\Pi$ determined by the points $\left(\mathbf{x}_{1} \wedge \ldots \wedge \mathbf{x}_{r}\right)\left(s_{i}^{\prime}\right)$. Since

$$
s_{i+1}^{\prime}-s_{i}^{\prime}=\frac{\left(s_{i+1}-s_{i}\right)+\ldots+\left(s_{i+r+1}-s_{i+r}\right)}{r+1} \leq|\Pi|,
$$

the length of $\Pi$ goes to the length of the curve $\left(\mathbf{x}_{1} \wedge \ldots \wedge \mathbf{x}_{r}\right)(s)$ as $|\Pi| \rightarrow 0$; in other words,

$$
\lim _{|\Pi| \rightarrow 0} \sum_{i=0}^{m-1} \beta_{i} \int_{C}\left\|\left(\mathbf{f}_{1} \wedge \ldots \wedge \mathbf{f}_{r}\right)^{\prime}(s)\right\| \mathrm{d} s
$$

where $\beta_{i}=\measuredangle\left(\left(\mathbf{f}_{1} \wedge \ldots \wedge \mathbf{f}_{r}\right)\left(s_{i}^{\prime}\right),\left(\mathbf{f}_{1} \wedge \ldots \wedge \mathbf{f}_{r}\right)\left(s_{i+1}^{\prime}\right)\right)$. Moreover, from the Frénet formulas (c.1)-(c.4) we deduce $\left(\mathbf{f}_{1} \wedge \ldots \wedge \mathbf{f}_{r}\right)^{\prime} \kappa_{r}\left(\mathbf{f}_{1} \wedge \ldots \wedge \mathbf{f}_{r-1} \wedge \mathbf{f}_{r+1}\right)$. Hence

$$
\lim _{|\Pi| \rightarrow 0} \sum_{i=0}^{m-1} \beta_{i} \int_{C}\left\|\kappa_{r}(s)\left(\mathbf{f}_{1} \wedge \ldots \wedge \mathbf{f}_{r-1} \wedge \mathbf{f}_{r+1}\right)(s)\right\| \mathrm{d} s=\int_{C}\left|\kappa_{r}(s)\right| \mathrm{d} s .
$$

Accordingly, it suffices to prove that $\lim _{|\Pi| \rightarrow 0} \sum_{i=0}^{m-1} \alpha_{i}=\lim _{|\Pi| \rightarrow 0} \sum_{i=0}^{m-1} \beta_{i}$. To do this, we consider the geodesic quadrilateral in $S\left(\bigwedge^{r} \mathbb{R}^{n}\right)$, the vertices of which are $\left(\left(\mathbf{f}_{1} \wedge \ldots \wedge \mathbf{f}_{r}\right)\left(s_{i}^{\prime}\right), \mathbf{u}_{i}, \mathbf{u}_{i+1},\left(\mathbf{f}_{1} \wedge \ldots \wedge \mathbf{f}_{r}\right)\left(s_{i+1}^{\prime}\right)\right)$ and the measures of its arcs are $\theta_{i}, \alpha_{i}, \theta_{i+1}, \beta_{i}$, cyclically. From the triangle inequality we obtain $\alpha_{i} \leq \theta_{i+1}+\beta_{i}+\theta_{i}$ and $\beta_{i} \leq \theta_{i}+\alpha_{i}+\theta_{i+1}$. Therefore, $\left|\alpha_{i}-\beta_{i}\right| \leq \theta_{i}+\theta_{i+1}$, and for every polygon $\Pi$ 
such that $|\Pi|<\delta$ from Lemma 2.2 we deduce

$$
\begin{aligned}
\left|\sum_{i=0}^{m-1} \alpha_{i}-\sum_{i=0}^{m-1} \beta_{i}\right| & \leq \sum_{i=0}^{m-1}\left|\alpha_{i}-\beta_{i}\right| \leq \sum_{i=0}^{m-1}\left(\theta_{i}+\theta_{i+1}\right)<\sum_{i=0}^{m-1}\left(\Delta_{r} s_{i}+\Delta_{r} s_{i+1}\right) \varepsilon \\
& =\varepsilon\left(\sum_{i=0}^{m-1}\left(s_{i+r}-s_{i}\right)+\sum_{i=0}^{m-1}\left(s_{i+r+1}-s_{i+1}\right)\right) \\
& =\varepsilon \sum_{i=0}^{m-1}\left(\left(s_{i+r}-s_{i+r-1}\right)+\ldots+\left(s_{i+1}-s_{i}\right)\right) \\
& +\varepsilon \sum_{i=0}^{m-1}\left(\left(s_{i+r+1}-s_{i+r}\right)+\ldots+\left(s_{i+2}-s_{i+1}\right)\right) \\
& =\varepsilon \sum_{i=0}^{m-1} \sum_{j=0}^{r-1}\left(s_{i+r-j}-s_{i+r-j-1}\right)+\varepsilon \sum_{i=0}^{m-1} \sum_{j=0}^{r-1}\left(s_{i+r+1-j}-s_{i+r-j}\right) \\
& =\varepsilon \sum_{j=0}^{r-1} \sum_{i=0}^{m-1}\left(\left(s_{i+r-j}-s_{i+r-j-1}\right)+\left(s_{i+r+1-j}-s_{i+r-j}\right)\right) \\
& \leq 2 r l \varepsilon
\end{aligned}
$$

thus proving Theorem 1.1 .

\section{REFERENCES}

1. A. D. Alexandrov and Yu. G. Reshetnyak, General Theory of Irregular Curves, Kluwer Academic Publishers, Dordrecht, The Netherlands, 1989. MR 92h:53003

2. S. Lang, Analysis I, Addison-Wesley Publishing Company, Reading, MA, 1969.

3. J. W. Milnor, On the total curvature of knots, Ann. of Math. 52, No. 2 (1950), 248-257. MR 12:273C

4. J. A. Wolf, Spaces of Constant Curvature, McGraw-Hill, Inc., NY, 1967. MR 36:829

Instituto de Física Aplicada, Consejo Superior de Investigaciones Cientificas, Calle Serrano 144, 28006-Madrid, Spain

Instituto de Física Aplicada, Consejo Superior de Investigaciones Cientificas, Calle SERRANO 144, 28006-MADRID, SPAIN

E-mail address: $\{$ luis, jaime\}@iec.csic.es 